This is a self-archived version of an original article. This version may differ from the original in pagination and typographic details.

Author(s): Blåsten, Eemeli; Lin, Yi-Hsuan

Title: Radiating and non-radiating sources in elasticity

Year: 2019

Version: Accepted version (Final draft)

Copyright: @ 2018 IOP Publishing Ltd

Rights: In Copyright

Rights url: http://rightsstatements.org/page/lnC/1.0/?language=en

Please cite the original version:

Blåsten, E., \& Lin, Y.-H. (2019). Radiating and non-radiating sources in elasticity. Inverse

Problems, 35(1), 015005. https://doi.org/10.1088/1361-6420/aae99e 


\title{
RADIATING AND NON-RADIATING SOURCES IN ELASTICITY
}

\author{
EEMELI BLÅSTEN AND YI-HSUAN LIN
}

\begin{abstract}
In this work, we study the inverse source problem of a fixed frequency for the Navier's equation. We investigate nonradiating external forces. If the support of such a force has a convex or non-convex corner or edge on their boundary, the force must be vanishing there. The vanishing property at corners and edges holds also for sufficiently smooth transmission eigenfunctions in elasticity. The idea originates from the enclosure method: an energy identity and a new type exponential solutions for the Navier's equation.
\end{abstract}

Keywords Inverse source problem, elastic waves, Navier's equation, exponential solutions, transmission eigenfunctions

Mathematics Subject Classification (2010): 35P25, 78A46, 74B05 (primary); 51M20 (secondary).

\section{Contents}

1. Introduction

1.1. Motivation and background

1.2. The mathematical formulation of elastic waves

2. Exponential solutions of the Navier's equation in the plane

3. Corner scattering

4. Proof of Theorems

5. Appendix

References

\section{INTRODUCTION}

1.1. Motivation and background. The inverse source problem is an important research topic in scattering theory. Its goal is to determine the shape of unknown sources by measuring the radiated wave patterns in the farfield. This problem is motivated by several scientific and industrial areas, such as medical imaging [21] and photo-acoustic tomography [2]. A classical example outside of elasticity is to utilize electric or magnetic fields on the periphery of the human body, such as in non-invasive brain image reconstruction. Mathematically, the inverse source problem for acoustic, electromagnetic and elastic waves has been studied widely by many researchers $[1,3,5,17,18,28,33]$. Furthermore, the inverse source problem can also be regarded as a basic mathematical method to study diffusion-based optical tomography, lidar imaging and fluorescence microscopy. 
The inverse source problem of a fixed frequency is ill-posed and its solution requires a-priori knowledge. These types of problems have picked interest before too, but for different equations. The umbrella term Schiffer's problem [16] captures the essence of these problems: from the knowledge of one far-field pattern, or the Cauchy data of one solution, determine the shape of a scatterer. [22] showed the unique determination of penetrable inclusions for the conductivity equation by the Cauchy boundary data of one solution, with reconstruction via the enclosure method appearing in [29]. In [28], the enclosure method is used to reconstruct the the convex hull of a polyhedral source or potential for the Helmholtz equation given a single measurement. In [30], the authors adapted the enclosure method for inverse obstacle problem by using a single set of the Cauchy data to the Navier's equation. Furthermore, in a slightly different context, the Schiffer's problem was studied in a nonlocal setup, see [14].

A new approach to this problem began from the realization that an acoustic potential that has a corner jump would scatter any incident wave at any wavenumbers [11]. This applies to the potential scattering setting without a source. However trying to apply it to source scattering produces an integration by parts formula used in the enclosure method [28]. The corner scattering method was then extended and used for the shape-determination of polyhedral potential scatterers [27], and to sources in [7]. The latter used a new type of complex geometrical optics solution and the enclosure method. These techniques also led to the surprising discovery that interior transmission eigenfunctions [15] vanish at convex corners [8,9]. For more corner and edge scattering results, we also refer readers to $[19,20]$. In this paper we extend the single measurement enclosure method technique to the elastic setting.

1.2. The mathematical formulation of elastic waves. Let $\lambda, \mu$ be the Lamé constants satisfying the following strong convexity condition

$$
\mu>0 \text { and } n \lambda+2 \mu>0, \text { for } n=2,3 .
$$

Let $\boldsymbol{f} \in \mathbb{C}^{n}$ be an external force, which is assumed to be compactly supported. More specifically, the function $\boldsymbol{f}=\chi_{\Omega} \boldsymbol{\varphi}$, where $\chi_{\Omega}$ is the characteristic function of a bounded Lipschitz domain $\Omega$ in $\mathbb{R}^{n}$ and $\varphi \in L^{\infty}\left(\mathbb{R}^{n} ; \mathbb{C}^{n}\right)$. Given an angular frequency $\omega>0$, let $\boldsymbol{u}(x)=\left(u_{\ell}(x)\right)_{\ell=1}^{n}$ be the displacement vector field. Then the time-harmonic elastic system is

$$
\lambda \Delta \boldsymbol{u}+(\lambda+\mu) \nabla \nabla \cdot \boldsymbol{u}+\omega^{2} \boldsymbol{u}=\boldsymbol{f} \text { in } \mathbb{R}^{n} .
$$

Via the well-known Helmholtz decomposition in $\mathbb{R}^{n} \backslash \bar{\Omega}$, one can see that the scattered field can be decomposed as

$$
\boldsymbol{u}=\boldsymbol{u}_{p}+\boldsymbol{u}_{s} \text { in } \mathbb{R}^{n} \backslash \bar{\Omega},
$$

with

$$
\boldsymbol{u}_{p}=-\frac{1}{\omega_{p}^{2}} \nabla(\nabla \cdot \boldsymbol{u}) \text { and } \boldsymbol{u}_{s}=\frac{1}{\omega_{s}^{2}} \operatorname{rot}(\operatorname{rot} \boldsymbol{u}),
$$

where $\omega_{p}$ and $\omega_{s}$ are the compressional and shear wave numbers, respectively, which are given by

$$
\omega_{p}=\frac{\omega}{\sqrt{\lambda+2 \mu}} \text { and } \omega_{s}=\frac{\omega}{\sqrt{\mu}} \text {. }
$$


Above rot $=\nabla^{\perp}$ represents $\frac{\pi}{2}$ clockwise rotation of the gradient when $n=2$, and rot $=\nabla \times$ stands for the curl operator when $n=3$. The vector fields $\boldsymbol{u}_{p}$ and $\boldsymbol{u}_{s}$ are called the compressional and shear parts of the scattered vector field $\boldsymbol{u}$, respectively. In addition, recall that $\boldsymbol{f}=0$ in $\mathbb{R}^{n} \backslash \bar{\Omega}$. Then $\boldsymbol{u}_{p}$ and $\boldsymbol{u}_{s}$ satisfy the Helmholtz equation

$$
\begin{aligned}
& \left(\Delta+\omega_{p}^{2}\right) \boldsymbol{u}_{p}=0 \text { and } \operatorname{rot} \boldsymbol{u}_{p}=0 \text { in } \mathbb{R}^{n} \backslash \bar{\Omega}, \\
& \left(\Delta+\omega_{s}^{2}\right) \boldsymbol{u}_{s}=0 \text { and } \nabla \cdot \boldsymbol{u}_{s}=0 \text { in } \mathbb{R}^{n} \backslash \bar{\Omega} .
\end{aligned}
$$

Therefore, for the elastic scattering problem of Equation (1.2), we need to pose the Kupradze radiation condition

$$
\lim _{r \rightarrow \infty}\left(\frac{\partial \boldsymbol{u}_{p}}{\partial r}-i \omega_{p} \boldsymbol{u}_{p}\right)=0 \text { and } \lim _{r \rightarrow \infty}\left(\frac{\partial \boldsymbol{u}_{s}}{\partial r}-i \omega_{s} \boldsymbol{u}_{s}\right)=0, \quad r=|x|,
$$

uniformly in all directions $\widehat{x}=x /|x|$. Moreover, one can also expand the functions $\boldsymbol{u}_{s}$ and $\boldsymbol{u}_{p}$ as

$$
\begin{aligned}
& \boldsymbol{u}_{s}(x)=\frac{1}{4 \pi} \frac{e^{i \omega_{s}|x|}}{|x|^{\frac{n-1}{2}}} \boldsymbol{u}_{s}^{\infty}(\widehat{x})+O\left(|x|^{-\frac{n+1}{2}}\right) \text { as }|x| \rightarrow \infty, \\
& \boldsymbol{u}_{p}(x)=\frac{1}{4 \pi} \frac{e^{i \omega_{p}|x|}}{|x|^{\frac{n-1}{2}}} \boldsymbol{u}_{p}^{\infty}(\widehat{x})+O\left(|x|^{-\frac{n+1}{2}}\right) \text { as }|x| \rightarrow \infty,
\end{aligned}
$$

for $n=2,3$, where $\boldsymbol{u}_{s}^{\infty}$ and $\boldsymbol{u}_{p}^{\infty}$ denote the transversal and longitudinal elastic far fields radiated by the source $\boldsymbol{f}$. Furthermore, $\boldsymbol{u}_{s}^{\infty}$ and $\boldsymbol{u}_{p}^{\infty}$ can be explicitly represented by

$$
\boldsymbol{u}_{s}^{\infty}(\boldsymbol{e})=\Pi_{\boldsymbol{e}^{\perp}}\left(\int_{\mathbb{R}^{n}} e^{-i \omega_{s} \boldsymbol{e} \cdot y} \boldsymbol{f}(y) d y\right), \boldsymbol{u}_{p}^{\infty}(\boldsymbol{e})=\Pi_{\boldsymbol{e}}\left(\int_{\mathbb{R}^{n}} e^{-i \omega_{p} \boldsymbol{e} \cdot y} \boldsymbol{f}(y) d y\right),
$$

for any unit vector $\boldsymbol{e} \in \mathbb{S}^{n-1}$, where $\Pi_{\boldsymbol{e}}$ is the projection operator with respect to $\boldsymbol{e}$. Notice that the vector fields $\boldsymbol{u}_{s}^{\infty}$ and $\boldsymbol{u}_{p}^{\infty}$ are the tangential and the normal components of the Fourier transform of $\boldsymbol{f}$ evaluated on $\mathbb{S}^{n-1}$. Note that the elastic far fields (1.5) of the Navier's equation are derived using the Helmholtz decomposition of Equation (1.2) and the far-field patterns for the Helmholtz equations of (1.3), which is allowed by the radiation conditions of Equation (1.4). For a more detailed discussion, we refer readers to $[24,25,26]$.

It is known that for a given source function $\boldsymbol{f} \in L^{\infty}\left(\Omega ; \mathbb{C}^{n}\right) \subset L^{2}\left(\Omega ; \mathbb{C}^{n}\right)$, the scattering problem of Equations (1.2) and (1.4) has a unique solution (see [4] for instance):

$$
\boldsymbol{u}(x ; \omega)=\int_{\Omega} \boldsymbol{G}(x, y ; \omega) \cdot \boldsymbol{f}(y) d y,
$$

where $\boldsymbol{G}(x, y ; \omega) \in \mathbb{C}^{n \times n}$ is the Green's tensor for the Navier's equation in Equation (1.2). More precisely, the Green's tensor can be expressed as

$$
\boldsymbol{G}(x, y ; \omega)=\frac{1}{\mu} G_{n}\left(x, y ; \omega_{s}\right) I_{n}+\frac{1}{\omega^{2}} \nabla_{x} \nabla_{x}^{\perp}\left(G_{n}\left(x, y ; \omega_{s}\right)-G_{n}\left(x, y ; \omega_{p}\right)\right),
$$


where $I_{n}$ denotes an $n \times n$ identity matrix and

$$
G_{n}(x, y ; \omega)= \begin{cases}\frac{i}{4} H_{0}^{(1)}(\omega|x-y|) & \text { when } n=2, \\ \frac{1}{4 \pi} \frac{e^{i \omega|x-y|}}{|x-y|} & \text { when } n=3,\end{cases}
$$

is the fundamental solution for the Helmholtz equation. Here $H_{0}^{(1)}$ is the Hankel function of the first kind with order zero.

We prove the following three theorems. The first one is for the inverse source problem. It states that if an external force is applied to a region having a corner or edge on an elastic body, it creates a propagating elastic wave at any wavenumber.

Theorem 1.1. Let $\boldsymbol{f}=\chi_{\Omega} \varphi$ for a bounded domain $\Omega \subset \mathbb{R}^{n}, n \in\{2,3\}$ and bounded vector function $\varphi \in L^{\infty}\left(\mathbb{R}^{n}\right)$. Let $\omega, \mu>0, n \lambda+2 \mu>0$ and $\boldsymbol{u} \in H_{\text {loc }}^{2}\left(\mathbb{R}^{n}\right)$ satisfy Equation (1.2) and the radiation condition of Equation (1.4).

Assume that $\Omega$ has a corner (2D) or an edge (3D) that can be connected to infinity by a path in $\mathbb{R}^{n} \backslash \bar{\Omega}$, and that $\varphi$ is Hölder-continuous near it. If $\boldsymbol{u}$ has zero far-field pattern, then $\boldsymbol{\varphi}=0$ on the corner or edge, i.e. $\boldsymbol{\varphi}$ is the zero vector. In other words, $f$ has no jumps at these locations.

Remark. Recall that by the Helmholtz decomposition, one can reduce the Navier's equation of (1.2) into two Helmholtz equations as in (1.3) outside of $\Omega$. One might wonder if it were possible to prove Theorem 1.1 using the same decomposition for the source term and using a version of Theorem 1.1 proven in [7] that holds for the two Helmholtz equations of (1.3) separately. After all, by the Helmholtz decomposition, one could split $f=f_{p}+f_{s}$ and rewrite Equation (1.2) into

$$
\left(\Delta+\omega_{p}^{2}\right) u_{p}=f_{p}, \text { and }\left(\Delta+\omega_{s}^{2}\right) u_{s}=f_{s} \text { in } \mathbb{R}^{n} .
$$

However there is a problem of smoothness. Theorem 1.1 assumes only that the function $f$ is Hölder-continuous near a corner. This does not guarantee that both of $f_{p}$ and $f_{s}$ are Hölder-continuous near the same corner. This prevents the use of [7, Theorem 1.1] to derive the same conclusion for the Navier's equation.

In order to prove Theorem 1.1, we need to construct a new type exponential solution for the Navier's equation in the plane. The classical exponential solutions for the Navier's equation is called the complex geometrical optics (CGO) solutions. As a matter of fact, the CGO solutions [31] were utilized to show global uniqueness for the inverse problem of the isotropic elasticity system with infinite measurements. In [31], the authors introduced CGO solutions for the isotropic elasticity system by decoupling the system into weakly coupled systems. Their principal part is an exponential of quadratic complex-valued function.

The second theorem shows source shape and boundary value determination from a single far-field pattern. 
Theorem 1.2. Let $n \in\{2,3\}$ and $\Omega, \Omega^{\prime} \subset \mathbb{R}^{n}$ be bounded convex polyhedral domains. Let $\varphi, \varphi^{\prime} \in C^{\alpha}\left(\mathbb{R}^{n}\right)$, for some $\alpha \in(0,1)$ and have nonzero value on $\partial \Omega, \partial \Omega^{\prime}$.

Define $\boldsymbol{f}=\chi_{\Omega} \boldsymbol{\varphi}, \boldsymbol{f}^{\prime}=\chi_{\Omega^{\prime}} \boldsymbol{\varphi}^{\prime}$. Let $\omega, \mu>0, n \lambda+2 \mu>0$ and $\boldsymbol{u}, \boldsymbol{u}^{\prime} \in$ $H_{\text {loc }}^{2}\left(\mathbb{R}^{n}\right)$ have elastic sources $\boldsymbol{f}, \boldsymbol{f}^{\prime}$. In other words they satisfy Equation (1.2) with the radiation condition of Equation (1.4).

If $\boldsymbol{u}$ and $\boldsymbol{u}^{\prime}$ have the same far-field pattern then $\Omega=\Omega^{\prime}$ and $\boldsymbol{\varphi}=\varphi^{\prime}$ at each of their vertices and in three dimensions, edges.

Strictly speaking, we do not need to have $\varphi, \varphi^{\prime}$ Hölder-continuous everywhere or non-vanishing on the whole boundary for the unique determination of the shape. Near the corners is enough. In three dimensions, if they are in addition non-vanishing and Hölder-continuous near the edges, then we can also deduce that $\varphi=\varphi^{\prime}$ on them.

Definition 1.3 (Interior transmission eigenfunctions). A pair $(\boldsymbol{v}, \boldsymbol{w}) \in$ $L^{2}(\Omega) \times L^{2}(\Omega)$ is called interior transmission eigenfunctions for the Navier equations with density $V \in L^{\infty}(\Omega)$ at the interior transmission eigenvalue $\omega \in \mathbb{R}_{+}$if

$$
\left\{\begin{array}{l}
\lambda \Delta \boldsymbol{w}+(\lambda+\mu) \nabla \nabla \cdot \boldsymbol{w}+\omega^{2} \boldsymbol{w}=0, \\
\lambda \Delta \boldsymbol{v}+(\lambda+\mu) \nabla \nabla \cdot \boldsymbol{v}+\omega^{2}(1+V) \boldsymbol{v}=0,
\end{array}\right.
$$

and $\boldsymbol{v}-\boldsymbol{w} \in H^{2}(\Omega)$ with $\boldsymbol{v}=\boldsymbol{w}$ and $\boldsymbol{T}_{\boldsymbol{\nu}} \boldsymbol{v}=\boldsymbol{T}_{\boldsymbol{\nu}} \boldsymbol{w}$ on $\partial \Omega$. Nothing is imposed on the boundary values of $\boldsymbol{v}, \boldsymbol{w}$ individually.

Above $\boldsymbol{T}_{\boldsymbol{\nu}}$ is the boundary tration operator.

Definition 1.4. The boundary traction operator $\boldsymbol{T}_{\boldsymbol{\nu}}$ is defined as follows. In the two-dimensional case it is

$$
\boldsymbol{T}_{\boldsymbol{\nu}} \boldsymbol{u}=2 \mu \frac{\partial \boldsymbol{u}}{\partial \boldsymbol{\nu}}+\lambda \boldsymbol{\nu} \nabla \cdot \boldsymbol{u}+\mu \boldsymbol{\nu}^{\perp}\left(\partial_{2} u_{1}-\partial_{1} u_{2}\right),
$$

where $\boldsymbol{\nu}=\left(\nu_{1}, \nu_{2}\right)$ is a unit outer normal on $\partial \Omega$ and $\boldsymbol{\nu}^{\perp}:=\left(-\nu_{2}, \nu_{1}\right)$. In the three dimensional case,

$$
\boldsymbol{T}_{\boldsymbol{\nu}} \boldsymbol{u}=2 \mu \frac{\partial \boldsymbol{u}}{\partial \boldsymbol{\nu}}+\lambda \boldsymbol{\nu} \nabla \cdot \boldsymbol{u}+\mu \boldsymbol{\nu} \times(\nabla \times \boldsymbol{u}),
$$

where $\boldsymbol{\nu}=\left(\nu_{1}, \nu_{2}, \nu_{3}\right)$.

The interior transmission problem is a well studied problem in inverse scattering theory. In particular the sampling method for solving the inverse scattering problem fails at wavenumbers that are transmission eigenvalues [16]. Notable results, $[12,15,36,38]$ and the recent survey [13], have so far focused mostly on the spectral properties of the transmission eigenvalues. Only recently, e.g. [10, 32, 37], have results about the eigenfunctions themselves started surfacing, in the form of completeness of the eigenfunctions in some sense. These past results are mostly in the context of acoustic scattering for the Helmholtz equation. [6] considers the fundamental properties in the context of elasticity, and shows the existence and discreteness of the transmission eigenvalues.

Recently in $[7,8,9]$ it was shown that under given smoothness and geometric assumptions the transmission eigenfunctions for the Helmholtz equation 
vanish at convex corners of the domain $\Omega$. We show the same conclusion for the interior transmission problem for an elastic material with varying density.

Theorem 1.5. Let $n \in\{2,3\}$ and $\Omega \subset \mathbb{R}^{n}$ be a bounded domain. Let $V \in$ $L^{\infty}(\Omega)$ be the material density, and $\mu>0, n \lambda+2 \mu>0$ be constant Lamé parameters. Assume that $\omega>0$ is an interior transmission eigenvalue and $\boldsymbol{v}, \boldsymbol{w} \in L^{2}(\Omega)$ are the corresponding transmission eigenfunctions defined by Equation (1.6).

Let $x_{c}$ be any vertex or edge of $\partial \Omega$ around which $V$ and either one of $\boldsymbol{v}, \boldsymbol{w}$ are $C^{\alpha}$ smooth, for some $\alpha \in(0,1)$. Then so is the other, and $\boldsymbol{v}\left(x_{c}\right)=$ $\boldsymbol{w}\left(x_{c}\right)=0$ if $V\left(x_{c}\right) \neq 0$.

The paper is structured as follows. In Section 2, we construct new type exponential solution for the two-dimensional isotropic elastic case and develop the integration by parts formula for the domain with corners. In Section 3, we discuss the corner scattering in a plane, and we use the dimensional reduction technique to solve the three-dimensional case. In addition, we prove our theorems in Section 4. Finally, in Section 5, we show that there is a nonradiating source $\boldsymbol{f}$ that generates elastic far fields that vanish in all directions.

\section{Exponential solutions of the NavieR's EQUation in the Plane}

We write $\mathcal{L}:=\lambda \Delta+(\lambda+\mu) \nabla(\nabla \cdot)$ for the second order elliptic operator. If $\boldsymbol{u}$ solves the elasticity system of Equation (1.2) then $\mathcal{L} u=\boldsymbol{f}$ in $\mathbb{R}^{n}$ for $n=2,3$, where $\boldsymbol{f}$ is the given compactly supported bounded source function. Next, let $\boldsymbol{v}$ be a solution of $\mathcal{L} \boldsymbol{v}=0$. Via the standard regularity theory for the second order elliptic system (see [35, Theorem 4.16] for instance), one can obtain that $u, v$ are $H_{l o c}^{2}\left(\mathbb{R}^{n}\right)$ functions. Then integrating by parts twice yields that

$$
\int_{\Omega} \boldsymbol{f} \cdot \boldsymbol{v} d x=\int_{\Omega}(\mathcal{L} \boldsymbol{u}) \cdot \boldsymbol{v} d x=\int_{\partial \Omega}\left[\left(\boldsymbol{T}_{\boldsymbol{\nu}} \boldsymbol{u}\right) \cdot \boldsymbol{v}-\left(\boldsymbol{T}_{\boldsymbol{\nu}} \boldsymbol{v}\right) \cdot \boldsymbol{u}\right] d S,
$$

where $\boldsymbol{T}_{\boldsymbol{\nu}}$ stands for the boundary traction operator (Definition 1.4) of Equation (1.2).

In two dimensions, note that the system of Equation (1.2) can be expressed componentwise as

$$
\mathcal{L} \boldsymbol{u}=\left(\begin{array}{cc}
\lambda \Delta+(\lambda+\mu) \partial_{1}^{2} & (\lambda+\mu) \partial_{1} \partial_{2} \\
(\lambda+\mu) \partial_{1} \partial_{2} & \lambda \Delta+(\lambda+\mu) \partial_{2}^{2}
\end{array}\right) \boldsymbol{u}=\boldsymbol{f} \text { in } \mathbb{R}^{2} .
$$

From now on we identify $\mathbb{R}^{2}$ with the complex plane $\mathbb{C}$, and we have the following lemma.

Lemma 2.1. Let $\Omega \subset \mathbb{C}$ such that $\Omega \cap\left(\mathbb{R}_{-} \cup\{0\}\right)=\emptyset$. Let

$$
\boldsymbol{v}(x)=\left(\begin{array}{c}
\exp (-s \sqrt{z}) \\
i \exp (-s \sqrt{z})
\end{array}\right)
$$

where $z=x_{1}+i x_{2}$ and $s \in \mathbb{R}_{+}$. The complex square root is defined as

$$
\sqrt{z}=\sqrt{|z|}\left(\cos \frac{\theta}{2}+i \sin \frac{\theta}{2}\right)
$$

where $-\pi<\theta \leq \pi$ is the argument of $z$. Then $\boldsymbol{v}$ satisfies $\mathcal{L} \boldsymbol{v}=0$ in $\Omega$. 
Proof. The complex square root is complex analytic in $\mathbb{C}$ except at the branch cut composed of the origin and the negative real axis. Thus the components of $\boldsymbol{v}=\left(v_{1}, v_{2}\right)^{T}$ are complex analytic in $\Omega$. Let us rewrite the differential operator in Equation (2.2) using the complex derivatives

$$
\partial=\frac{1}{2}\left(\partial_{1}-i \partial_{2}\right), \quad \bar{\partial}=\frac{1}{2}\left(\partial_{1}+i \partial_{2}\right) .
$$

These imply that $\Delta=4 \partial \bar{\partial}$ and that $\partial_{1}=\partial+\bar{\partial}, \partial_{2}=i(\partial-\bar{\partial})$. Hence the abovementioned operator becomes

$$
\mathcal{L}=\left(\begin{array}{cc}
4 \lambda \partial \bar{\partial}+(\lambda+\mu)\left(\partial^{2}+2 \partial \bar{\partial}+\bar{\partial}^{2}\right) & (\lambda+\mu) i\left(\partial^{2}-\bar{\partial}^{2}\right) \\
(\lambda+\mu) i\left(\partial^{2}-\bar{\partial}^{2}\right) & 4 \lambda \partial \bar{\partial}-(\lambda+\mu)\left(\partial^{2}-2 \partial \bar{\partial}+\bar{\partial}^{2}\right)
\end{array}\right)
$$

and thus $\mathcal{L} v=0$ if and only if

$$
\left\{\begin{array}{l}
4 \lambda \partial \bar{\partial} v_{1}+(\lambda+\mu)\left(\partial^{2} v_{1}+2 \partial \bar{\partial} v_{1}+\bar{\partial}^{2} v_{1}+i \partial^{2} v_{2}-i \bar{\partial}^{2} v_{2}\right)=0 \\
4 \lambda \partial \bar{\partial} v_{2}+(\lambda+\mu)\left(i \partial^{2} v_{1}-i \bar{\partial}^{2} v_{1}-\partial^{2} v_{2}+2 \partial \bar{\partial} v_{2}-\bar{\partial}^{2} v_{2}\right)=0 .
\end{array}\right.
$$

Recall that our choice of $\boldsymbol{v}$ implies that $\bar{\partial} v_{1}=\bar{\partial} v_{2}=0$ in $\Omega$. Hence the above reduces to

$$
\partial^{2} v_{1}+i \partial^{2} v_{2}=0, \quad i \partial^{2} v_{1}-\partial^{2} v_{2}=0
$$

which is just equivalent to $\partial^{2} v_{1}+i \partial^{2} v_{2}=0$. But this is true since $v_{1}+i v_{2}=0$. Hence $\mathcal{L} \boldsymbol{v}=0$ in $\Omega$.

The following lemma provides the integration by parts formula around the corner, where $\boldsymbol{v}$ is not smooth.

Lemma 2.2. Let $\boldsymbol{v}: \mathbb{R}^{2} \rightarrow \mathbb{C}^{2}$ be defined by (2.3) from Lemma 2.1 and

$$
\mathcal{K}=\left\{x \in \mathbb{R}^{2} \mid x \neq 0, \theta_{m}<\arg \left(x_{1}+i x_{2}\right)<\theta_{M}\right\}
$$

for given angles $-\pi<\theta_{m}<\theta_{M}<\pi$. Assume that $\boldsymbol{u} \in H^{2}\left(\mathcal{K} \cap B ; \mathbb{C}^{2}\right)$ where $B=B(0, h)$ for some $h>0$. Moreover let $\boldsymbol{u}=\boldsymbol{T}_{\boldsymbol{\nu}} \boldsymbol{u}=0$ in $B \cap \partial \mathcal{K}$. Then

$$
\int_{\mathcal{K} \cap B} \boldsymbol{v} \cdot \mathcal{L} \boldsymbol{u} d x=\int_{\mathcal{K} \cap \partial B}\left[\left(\boldsymbol{T}_{\boldsymbol{\nu}} \boldsymbol{u}\right) \cdot \boldsymbol{v}-\left(\boldsymbol{T}_{\boldsymbol{\nu}} \boldsymbol{v}\right) \cdot \boldsymbol{u}\right] d S
$$

Proof. Let $\mathcal{K}_{\varepsilon}:=(\mathcal{K} \cap B) \backslash B(0, \varepsilon)$ for $0<\varepsilon<h$. Note that $|\boldsymbol{v}|$ is bounded and $|\mathcal{L} \boldsymbol{u}| \in L^{2}$, and that $\boldsymbol{v}$ is smooth and $\mathcal{L} \boldsymbol{v}=0$ in the closure of $\mathcal{K}_{\varepsilon}$. Hence we have

$$
\int_{\mathcal{K} \cap B} \boldsymbol{v} \cdot \mathcal{L} \boldsymbol{u} d x=\lim _{\varepsilon \rightarrow 0} \int_{\mathcal{K}_{\varepsilon}} \boldsymbol{v} \cdot \mathcal{L} \boldsymbol{u} d x=\lim _{\varepsilon \rightarrow 0} \int_{\partial \mathcal{K}_{\varepsilon}}\left[\left(\boldsymbol{T}_{\boldsymbol{\nu}} \boldsymbol{u}\right) \cdot \boldsymbol{v}-\left(\boldsymbol{T}_{\boldsymbol{\nu}} \boldsymbol{v}\right) \cdot \boldsymbol{u}\right] d S
$$

by Equation (2.1). Recall that $\boldsymbol{u}=\boldsymbol{T}_{\boldsymbol{\nu}} \boldsymbol{u}=0$ on $B \cap \partial \mathcal{K}$. Thus the boundary integral of Equation (2.7) is equal to

$$
\int_{\mathcal{K} \cap \partial B}\left[\left(\boldsymbol{T}_{\boldsymbol{\nu}} \boldsymbol{u}\right) \cdot \boldsymbol{v}-\left(\boldsymbol{T}_{\boldsymbol{\nu}} \boldsymbol{v}\right) \cdot \boldsymbol{u}\right] d S+\int_{\mathcal{K} \cap S(0, \varepsilon)}\left[\left(\boldsymbol{T}_{\boldsymbol{\nu}} \boldsymbol{u}\right) \cdot \boldsymbol{v}-\left(\boldsymbol{T}_{\boldsymbol{\nu}} \boldsymbol{v}\right) \cdot \boldsymbol{u}\right] d S,
$$

where $S(0, \epsilon)=\partial B(0, \epsilon)$, and so our task is to show that the last integral in Equation (2.8) vanishes as $\varepsilon \rightarrow 0$.

Notice that the normal derivative is $\boldsymbol{\nu}(x)=-x /|x|$ on $S(0, \varepsilon)$. Recall also that $\partial_{1}=\partial+\bar{\partial}$ and $\partial_{2}=i(\partial-\bar{\partial})$, and moreover that $\boldsymbol{v}$ is complex analytic 
in a neighbourhood of $S(0, \varepsilon)$. Hence $\partial_{1} \boldsymbol{v}=\partial \boldsymbol{v}$ and $\partial_{2} \boldsymbol{v}=i \partial \boldsymbol{v}$. Concluding, the boundary traction becomes

$$
\begin{aligned}
\boldsymbol{T}_{\boldsymbol{\nu}} \boldsymbol{v} & =2 \mu\left(\nu_{1} \partial_{1}+\nu_{2} \partial_{2}\right) \boldsymbol{v}+\lambda \boldsymbol{\nu}\left(\partial_{1} v_{1}+\partial_{2} v_{2}\right)+\mu \boldsymbol{\nu}^{\perp}\left(\partial_{2} v_{1}-\partial_{1} v_{2}\right) \\
& =2 \mu\left(\nu_{1}+i \nu_{2}\right) \partial \boldsymbol{v}+\lambda \boldsymbol{\nu}\left(\partial v_{1}+i \partial v_{2}\right)+\mu \boldsymbol{\nu}^{\perp}\left(i \partial v_{1}-\partial v_{2}\right),
\end{aligned}
$$

however note that $v_{2}=i v_{1}$ so the divergence and curl terms vanish. Moreover $\nu_{1}+i \nu_{2}=-z /|z|$ with the identification $z=x_{1}+i x_{2}$. Hence

$$
\boldsymbol{T}_{\boldsymbol{\nu}} \boldsymbol{v}=-2 \mu \frac{z}{|z|} \partial \boldsymbol{v}=s \mu \frac{\sqrt{z}}{|z|} \boldsymbol{v}
$$

because $\partial \exp (-s \sqrt{z})=\frac{-s \exp (-s \sqrt{z})}{2 \sqrt{z}}$ outside of the branch cut.

We have $|\boldsymbol{v}(x)|=\sqrt{2} \exp (-s \sqrt{|x|} \cos \theta / 2) \leq \sqrt{2}$ where $\theta$ is the argument of $x_{1}+i x_{2}$. By Equation (2.9)

$$
\left|\boldsymbol{T}_{\nu} \boldsymbol{v}\right|=\left|s \mu \frac{\sqrt{z}}{|z|} \boldsymbol{v}\right|=s \mu \varepsilon^{-1 / 2}|\boldsymbol{v}| \leq \sqrt{2} s \mu \varepsilon^{-1 / 2}
$$

on $S(0, \varepsilon)$. Note also that $H^{2}(\mathcal{K} \cap B)$-functions embed continuously into uniformly bounded and continuous functions in two dimensions. Hence

$$
|\boldsymbol{u}(x)| \leq C\|\boldsymbol{u}\|_{H^{2}(\mathcal{K} \cap B)}
$$

for $x \in \mathcal{K} \cap B$ and in particular for $x \in S(0, \varepsilon)$. This shows that

$$
\left|\int_{\mathcal{K} \cap S(0, \varepsilon)}\left(\boldsymbol{T}_{\boldsymbol{\nu}} \boldsymbol{v}\right) \cdot \boldsymbol{u}(x) d S\right| \leq C\|\boldsymbol{u}\|_{H^{2}(\mathcal{K} \cap B)} \sqrt{2} s \varepsilon^{-1 / 2}\left(\theta_{M}-\theta_{m}\right) \varepsilon \rightarrow 0
$$

as $\varepsilon \rightarrow 0$.

For the remaining term, we can estimate $|\boldsymbol{v}| \leq \sqrt{2}$ and use the CauchySchwarz inequality to get

$$
\left|\int_{\mathcal{K} \cap S(0, \varepsilon)}\left(\boldsymbol{T}_{\boldsymbol{\nu}} \boldsymbol{u}\right) \cdot \boldsymbol{v} d S\right| \leq \sqrt{\left(\theta_{M}-\theta_{m}\right) \varepsilon}\left\|\boldsymbol{T}_{\boldsymbol{\nu}} \boldsymbol{u}\right\|_{L^{2}(\mathcal{K} \cap S(0, \varepsilon))} \sqrt{2} .
$$

Denote $\boldsymbol{g}(x)=\boldsymbol{T}_{\boldsymbol{\nu}} \boldsymbol{u}(x)$. Then $\boldsymbol{g} \in H^{1}(K \cap B)$ and $\|\boldsymbol{g}\|_{H^{1}} \leq C(\lambda+\mu)\|\boldsymbol{u}\|_{H^{2}}$ in any given open set. Let $\boldsymbol{g}_{\varepsilon}(y)=\boldsymbol{g}(\varepsilon y)$. Then $d S(x)=\varepsilon d S(y)$ so

$$
\|\boldsymbol{g}\|_{L^{2}(\mathcal{K} \cap S(0, \varepsilon))}=\sqrt{\varepsilon}\left\|\boldsymbol{g}_{\varepsilon}\right\|_{L^{2}(\mathcal{K} \cap S(0,1))} \leq C \sqrt{\varepsilon}\left\|\boldsymbol{g}_{\varepsilon}\right\|_{H^{1}(\mathcal{K} \cap B(0,1))}
$$

by the trace theorem, and $C>0$ is independent of $\varepsilon$. However $d x=\varepsilon^{2} d y$, and $\partial_{y_{j}} \boldsymbol{g}_{\varepsilon}(y)=\partial_{y_{j}}(\boldsymbol{g}(\varepsilon y))=\varepsilon\left(\partial_{j} \boldsymbol{g}\right)(\varepsilon y)$ so

$$
\begin{aligned}
& \left\|\boldsymbol{g}_{\varepsilon}\right\|_{L^{2}(\mathcal{K} \cap B(0,1))}=\varepsilon^{-1}\|\boldsymbol{g}\|_{L^{2}(\mathcal{K} \cap B(0, \varepsilon))}, \\
& \left\|\partial_{j} \boldsymbol{g}_{\varepsilon}\right\|_{L^{2}(\mathcal{K} \cap B(0,1))}=\left\|\partial_{j} \boldsymbol{g}\right\|_{L^{2}(\mathcal{K} \cap B(0, \varepsilon))},
\end{aligned}
$$

in other words $\left\|\boldsymbol{g}_{\varepsilon}\right\|_{H^{1}(K \cap B(0,1))} \leq \varepsilon^{-1}\|\boldsymbol{g}\|_{H^{1}(K \cap B(0, \varepsilon))}$. This implies

$$
\|\boldsymbol{g}\|_{L^{2}(\mathcal{K} \cap S(0, \varepsilon))} \leq C \varepsilon^{-1 / 2}\|\boldsymbol{g}\|_{H^{1}(\mathcal{K} \cap B(0, \varepsilon))} \leq C(\lambda+\mu) \varepsilon^{-1 / 2}\|\boldsymbol{u}\|_{H^{2}(\mathcal{K} \cap B(0, \varepsilon))}
$$

where $C$ is independent of $\varepsilon$. Combining these with (2.10) gives

$$
\left|\int_{\mathcal{K} \cap S(0, \varepsilon)}\left(\boldsymbol{T}_{\boldsymbol{\nu}} \boldsymbol{u}\right) \cdot \boldsymbol{v} d S\right| \leq C(\lambda+\mu) \sqrt{\theta_{M}-\theta_{m}}\|\boldsymbol{u}\|_{H^{2}(\mathcal{K} \cap B(0, \varepsilon))}
$$


which tends to zero when $\varepsilon \rightarrow 0$ because $\|\boldsymbol{u}\|_{H^{2}(K \cap B)}$ is finite. This proves Equation (2.6).

\section{Corner SCATtERing}

Proposition 3.1. Let $\boldsymbol{v}: \mathbb{R}^{2} \rightarrow \mathbb{C}$ be the function given in Lemma 2.1 and define the open sector

$$
\mathcal{K}=\left\{x \in \mathbb{R}^{2} \mid x \neq 0, \theta_{m}<\arg \left(x_{1}+i x_{2}\right)<\theta_{M}\right\}
$$

for angles satisfying $-\pi<\theta_{m}<\theta_{M}<\pi$. Then

$$
\int_{\mathcal{K}} v_{1}(x) d x=6 i\left(e^{-2 \theta_{M} i}-e^{-2 \theta_{m} i}\right) s^{-4} .
$$

In addition for $\alpha, h>0$ and $j \in\{1,2\}$ we have the upper bounds

$$
\int_{\mathcal{K}}\left|v_{j}(x)\right||x|^{\alpha} d x \leq \frac{2\left(\theta_{M}-\theta_{m}\right) \Gamma(2 \alpha+4)}{\delta_{\mathcal{K}}^{2 \alpha+4}} s^{-2 \alpha-4}
$$

and

$$
\int_{\mathcal{K} \backslash B(0, h)}\left|v_{j}(x)\right| d x \leq \frac{6\left(\theta_{M}-\theta_{m}\right)}{\delta_{\mathcal{K}}^{4}} s^{-4} e^{-\delta_{\mathcal{K}} s \sqrt{h} / 2} .
$$

where $\delta_{\mathcal{K}}=\min _{\theta_{m}<\theta<\theta_{M}} \cos (\theta / 2)$ is a positive constant.

Proof. This result is in [7, Section 2], however note that the parameter $s$ is outside of the square root in this article.

Proposition 3.2. Let $\Omega \subset \mathbb{R}^{2}$ be a bounded domain and define the cone

$$
\mathcal{K}=\left\{x \in \mathbb{R}^{2} \mid x \neq 0, \theta_{m}<\arg \left(x_{1}+i x_{2}\right)<\theta_{M}\right\}
$$

with angles $-\pi<\theta_{m}<\theta_{M}<\pi$ where $\theta_{M} \neq \theta_{m}+\pi$. Assume that $0 \in \partial \Omega$ is the centre of a ball $B$ for which $\Omega \cap B=\mathcal{K} \cap B$.

Given $\alpha \in(0,1)$ and $\boldsymbol{f} \in C^{\alpha}(\overline{\Omega \cap B})$, let $\boldsymbol{u} \in H^{2}(\Omega \cap B)$ solve

$$
\lambda \Delta \boldsymbol{u}+(\lambda+\mu) \nabla \nabla \cdot \boldsymbol{u}+\omega^{2} \boldsymbol{u}=\boldsymbol{f} \text { in } \Omega \cap B,
$$

for some fixed $\omega>0$. If $\boldsymbol{u}=0$ and $\boldsymbol{T}_{\boldsymbol{\nu}} \boldsymbol{u}=0$ on $\partial \Omega \cap B$ then $\boldsymbol{f}(0)=0$.

Proof. Write the equation as $\mathcal{L} \boldsymbol{u}+\omega^{2} \boldsymbol{u}=\boldsymbol{f}$ where $\mathcal{L}$ is as in Equation (2.2). We may assume that $\boldsymbol{f}$ is real-valued. If not, then split it and $\boldsymbol{u}$ into their real and imaginary parts $\boldsymbol{f}_{R}, \boldsymbol{f}_{I}, \boldsymbol{u}_{R}, \boldsymbol{u}_{I}$ which would then satisfy

$$
\begin{aligned}
& \mathcal{L} \boldsymbol{u}_{R}+\omega^{2} \boldsymbol{u}_{R}=\boldsymbol{f}_{R}, \\
& \mathcal{L} \boldsymbol{u}_{I}+\omega^{2} \boldsymbol{u}_{I}=\boldsymbol{f}_{I},
\end{aligned}
$$

with $\boldsymbol{u}_{R}=0=\boldsymbol{u}_{I}, \boldsymbol{T}_{\boldsymbol{\nu}} \boldsymbol{u}_{R}=0=\boldsymbol{T}_{\boldsymbol{\nu}} \boldsymbol{u}_{I}$ on $\partial \Omega \cap B$. Then once we show that $\boldsymbol{f}_{R}(0)=\boldsymbol{f}_{I}(0)=0$ this would imply the original claim of $\boldsymbol{f}(0)=0$.

Let $\boldsymbol{v}$ be as in Lemma 2.1 and so $\mathcal{L} \boldsymbol{v}=0$ in $\Omega \cap B$. Let $\tilde{\boldsymbol{f}}=\boldsymbol{f}-\omega^{2} \boldsymbol{u}$ and keep in mind that $\widetilde{\boldsymbol{f}}(0)=\boldsymbol{f}(0)$ because $\boldsymbol{u}=0$ on $\partial \Omega \cap B$. Lemma 2.2 implies that

$$
\int_{\mathcal{K} \cap B} \boldsymbol{v} \cdot \widetilde{\boldsymbol{f}} d x=\int_{\mathcal{K} \cap \partial B}\left[\left(\boldsymbol{T}_{\boldsymbol{\nu}} \boldsymbol{u}\right) \cdot \boldsymbol{v}-\left(\boldsymbol{T}_{\boldsymbol{\nu}} \boldsymbol{v}\right) \cdot \boldsymbol{u}\right] d S
$$

and moreover the left-hand side of Equation (3.2) can be split as

$$
\int_{\mathcal{K} \cap B} \boldsymbol{v} \cdot \widetilde{\boldsymbol{f}} d x=\int_{\mathcal{K}} \boldsymbol{v} \cdot \boldsymbol{f}(0) d x-\int_{\mathcal{K} \backslash B} \boldsymbol{v} \cdot \boldsymbol{f}(0) d x+\int_{\mathcal{K} \cap B} \boldsymbol{v} \cdot(\tilde{\boldsymbol{f}}-\tilde{\boldsymbol{f}}(0)) d x
$$


because $\tilde{\boldsymbol{f}}(0)=\boldsymbol{f}(0)$.

As in the proof of Lemma 2.2, we have $\boldsymbol{T}_{\boldsymbol{\nu}} \boldsymbol{v}=-s \mu\left(\frac{\sqrt{z}}{|z|}\right) \boldsymbol{v}$ on $\mathcal{K} \cap \partial B$ and so $\left|\boldsymbol{T}_{\boldsymbol{\nu}} \boldsymbol{v}\right|=s \mu h^{-1 / 2} \sqrt{2} \exp (-s \sqrt{h} \cos (\theta / 2))$ on $\mathcal{K} \cap \partial B$ where $h$ is the radius of $B$ and $\theta$ the argument of $x_{1}+i x_{2}$. Let $\delta_{\mathcal{K}}:=\min _{\theta_{m}<\theta<\theta_{M}} \cos (\theta / 2)$ and it is positive. Hence

$$
\left|\boldsymbol{T}_{\boldsymbol{\nu}} \boldsymbol{v}(x)\right| \leq C_{\mathcal{K}, B, \mu} s \exp \left(-\delta_{\mathcal{K}} s \sqrt{h}\right)
$$

for some positive constant $C_{\mathcal{K}, B, \mu}$ (depending on $\mathcal{K}, B$ and $\mu$ ), when $x \in$ $\mathcal{K} \cap \partial B$. By a similar consideration

$$
|\boldsymbol{v}(x)| \leq \sqrt{2} \exp \left(-\delta_{\mathcal{K}} s \sqrt{h}\right)
$$

on the same part of the boundary. Thus, Equation (3.4), Equation (3.5), the Cauchy-Schwarz and trace inequalities give that

$$
\begin{aligned}
& \left|\int_{\mathcal{K} \cap \partial B}\left[\left(\boldsymbol{T}_{\boldsymbol{\nu}} \boldsymbol{u}\right) \cdot \boldsymbol{v}-\left(\boldsymbol{T}_{\boldsymbol{\nu}} \boldsymbol{v}\right) \cdot \boldsymbol{u}\right] d S\right| \\
& \quad \leq C_{\mathcal{K}, B, \mu, \lambda}\|\boldsymbol{u}\|_{H^{2}(\mathcal{K} \cap B)}(1+s) \exp \left(-\delta_{\mathcal{K}} s \sqrt{h}\right)
\end{aligned}
$$

which decays exponentially as $s \rightarrow \infty$.

Let us estimate the three terms in Equation (3.3) next. But before that, we recall $\widetilde{\boldsymbol{f}}=\boldsymbol{f}-\omega^{2} \boldsymbol{u}$, and that $\boldsymbol{u} \in H^{2}$ which embeds continuously into $C^{\alpha}$ in two dimensions. Hence $\widetilde{\boldsymbol{f}} \in C^{\alpha}(\overline{K \cap B})$. Then Proposition 3.1 gives

$$
\begin{gathered}
\int_{\mathcal{K}} \boldsymbol{v} \cdot \boldsymbol{f}(0) d x=\left(f_{1}(0)+i f_{2}(0)\right) \int_{\mathcal{K}} v_{1}(x) d x=C_{\mathcal{K}}\left(f_{1}(0)+i f_{2}(0)\right) s^{-4} \\
\int_{\mathcal{K} \backslash B}|\boldsymbol{v} \cdot \boldsymbol{f}(0)| d x \leq C_{\mathcal{K}}^{\prime}\|\boldsymbol{f}\|_{L^{\infty}} s^{-4} \exp \left(-\delta_{\mathcal{K}} s \sqrt{h} / 2\right) \\
\int_{\mathcal{K} \cap B}|\boldsymbol{v} \cdot(\tilde{\boldsymbol{f}}-\tilde{\boldsymbol{f}}(0))| d x \leq\|\widetilde{\boldsymbol{f}}\|_{C^{\alpha}} \sum_{j=1}^{2} \int_{\mathcal{K} \cap B}\left|v_{j}(x)\right||x|^{\alpha} d x \\
\leq C_{\mathcal{K}, \alpha}\|\tilde{\boldsymbol{f}}\|_{C^{\alpha}} s^{-2 \alpha-4}
\end{gathered}
$$

where $C_{\mathcal{K}} \neq 0$ and the other constants $C_{\mathcal{K}}^{\prime}, C_{\mathcal{K}, \alpha}$ are finite and positive. Multiplying Equations (3.2) and (3.3) by $s^{4}$ and letting $s \rightarrow \infty$ implies that

$$
C_{\mathcal{K}}\left(f_{1}(0)+i f_{2}(0)\right)=0
$$

and since $\boldsymbol{f}$ is real-valued and $C_{\mathcal{K}} \neq 0$, that $\boldsymbol{f}(0)=0$.

Lemma 3.3 (Dimension reduction). Let $D$ be a locally Lipschitz open set in $\mathbb{R}^{2}, M>0$ and $\alpha \in(0,1)$ be constants. Given $\boldsymbol{f} \in C^{\alpha}\left(\bar{D} \times[-L, L] ; \mathbb{C}^{3}\right)$, let $\boldsymbol{u} \in H^{2}\left(D \times(-L, L) ; \mathbb{C}^{3}\right)$ be a solution of

$$
\begin{cases}\mathcal{L} \boldsymbol{u}(x)=\boldsymbol{f}(x), & \text { for } x=\left(x^{\prime}, x_{3}\right) \in D \times(-L, L), \\ \boldsymbol{u}(x)=0, \boldsymbol{T}_{\boldsymbol{\nu}} \boldsymbol{u}(x)=0 & \text { for } x=\left(x^{\prime}, x_{3}\right) \in \Gamma \times(-L, L),\end{cases}
$$

where $\Gamma \subset \partial D$ consists of two connected segments and $\mu>0,3 \lambda+2 \mu>0$. Consider $\phi \in C_{c}^{\infty}(-L, L)$ and $\xi \in \mathbb{R}$, and we define the dimension reduction operator $\boldsymbol{R}_{\xi}$ by

$$
\boldsymbol{R}_{\xi} g\left(x^{\prime}\right):=\int_{-L}^{L} e^{-i x_{3} \xi} \phi\left(x_{3}\right) g\left(x^{\prime}, x_{3}\right) d x_{3}, \text { for } x^{\prime} \in D .
$$


Then one has $\boldsymbol{R}_{\xi} \boldsymbol{u} \in H^{2}\left(D ; \mathbb{C}^{3}\right) \cap C^{\alpha}\left(D ; \mathbb{C}^{3}\right)$ and there is a function $\boldsymbol{F}_{\xi}=$ $\boldsymbol{F}_{\xi}\left(x^{\prime}\right) \in C^{\alpha}\left(D ; \mathbb{C}^{3}\right)$ such that $\boldsymbol{R}_{\xi} \boldsymbol{u}$ is a solution of

$$
\begin{cases}\widetilde{\mathcal{L}}\left(\boldsymbol{R}_{\xi} \boldsymbol{u}\left(x^{\prime}\right)\right)=\boldsymbol{F}_{\xi}\left(x^{\prime}\right) & \text { for } x^{\prime} \in D \\ \boldsymbol{R}_{\xi} u\left(x^{\prime}\right)=0, \boldsymbol{T}_{\boldsymbol{\nu}}\left(\boldsymbol{R}_{\xi} \boldsymbol{u}\right)=0 & \text { for } x^{\prime} \in \Gamma\end{cases}
$$

where

$$
\widetilde{\mathcal{L}}:=\left(\begin{array}{ccc}
\lambda \Delta^{\prime}+(\lambda+\mu) \partial_{1}^{2} & (\lambda+\mu) \partial_{1} \partial_{2} & 0 \\
(\lambda+\mu) \partial_{1} \partial_{2} & \lambda \Delta^{\prime}+(\lambda+\mu) \partial_{2}^{2} & 0 \\
0 & 0 & \lambda \Delta^{\prime}
\end{array}\right)
$$

with $\Delta^{\prime}:=\partial_{1}^{2}+\partial_{2}^{2}$ being the Laplace operator with respect to the $x^{\prime}$-variables. Furthermore, we have

$$
\boldsymbol{F}_{\xi}\left(x^{\prime}\right)=\boldsymbol{R}_{\xi} f\left(x^{\prime}\right) \text { for } x^{\prime} \in \Gamma .
$$

Proof. Denote $\boldsymbol{u}=\left(u_{\ell}\right)_{\ell=1}^{3}$, by using [7, Lemma 3.4], then one can conclude that $\boldsymbol{R}_{\xi} u_{\ell} \in H^{2}(D) \cap C^{\alpha}(D)$ for $\ell=1,2,3$. Hence, it remains to show that $\boldsymbol{R}_{\xi} \boldsymbol{u}$ solves Equation (3.7).

In order to derive the equation for $\boldsymbol{R}_{\xi} \boldsymbol{u}$, note that in the three-dimensional case, the isotropic elastic operator $\mathcal{L}$ can be rewritten as

$$
\mathcal{L}=\left(\begin{array}{ccc}
\lambda \Delta+(\lambda+\mu) \partial_{1}^{2} & (\lambda+\mu) \partial_{1} \partial_{2} & (\lambda+\mu) \partial_{1} \partial_{3} \\
(\lambda+\mu) \partial_{1} \partial_{2} & \lambda \Delta+(\lambda+\mu) \partial_{2}^{2} & (\lambda+\mu) \partial_{2} \partial_{3} \\
(\lambda+\mu) \partial_{1} \partial_{3} & (\lambda+\mu) \partial_{2} \partial_{3} & \lambda \Delta+(\lambda+\mu) \partial_{3}^{2}
\end{array}\right)
$$

then we also have $\widetilde{\mathcal{L}} \boldsymbol{u}=\boldsymbol{f}-\boldsymbol{h}(\boldsymbol{u})$, where

$$
\boldsymbol{h}(\boldsymbol{u})=\left(\begin{array}{c}
\lambda \partial_{3}^{2} u_{1}+(\lambda+\mu) \partial_{3} \partial_{1} u_{3} \\
\lambda \partial_{3}^{2} u_{2}+(\lambda+\mu) \partial_{3} \partial_{2} u_{3} \\
(2 \lambda+\mu) \partial_{3}^{2} u_{3}+(\lambda+\mu) \partial_{3}\left(\partial_{1} u_{1}+\partial_{2} u_{2}\right)
\end{array}\right) .
$$

The Lebesuge dominated convergence theorem and an integration by parts formula yield that

$$
\widetilde{\mathcal{L}}\left(\boldsymbol{R}_{\xi} \boldsymbol{u}\right)=\boldsymbol{F}_{\xi}\left(x^{\prime}\right):=\boldsymbol{R}_{\xi} \boldsymbol{f}\left(x^{\prime}\right)+I_{\xi}\left(x^{\prime}\right)+I I_{\xi}\left(x^{\prime}\right),
$$

where

$$
\begin{aligned}
I_{\xi}\left(x^{\prime}\right)= & -\int_{-L}^{L} e^{-i x_{3} \xi} \phi^{\prime \prime}\left(x_{3}\right)\left(\begin{array}{c}
\lambda u_{1} \\
\lambda u_{2} \\
(2 \lambda+\mu) u_{3}
\end{array}\right)\left(x^{\prime}, x_{3}\right) d x_{3} \\
& +2 i \xi \int_{-L}^{L} e^{-i x_{3} \xi} \phi^{\prime}\left(x_{3}\right)\left(\begin{array}{c}
\lambda u_{1} \\
\lambda u_{2} \\
(2 \lambda+\mu) u_{3}
\end{array}\right)\left(x^{\prime}, x_{3}\right) d x_{3} \\
& +\xi^{2} \boldsymbol{R}_{\xi}\left(\begin{array}{c}
\lambda u_{1} \\
\lambda u_{2} \\
(2 \lambda+\mu) u_{3}
\end{array}\right)\left(x^{\prime}\right)
\end{aligned}
$$


and

$$
\begin{aligned}
I I_{\xi}\left(x^{\prime}\right)= & -i \xi(\lambda+\mu) \boldsymbol{R}_{\xi}\left(\begin{array}{c}
\partial_{1} u_{3} \\
\partial_{2} u_{3} \\
\partial_{1} u_{1}+\partial_{2} u_{2}
\end{array}\right)\left(x^{\prime}\right) \\
& +(\lambda+\mu) \int_{-L}^{L} e^{-i x_{3} \xi} \phi^{\prime}\left(x_{3}\right)\left(\begin{array}{c}
\partial_{1} u_{3} \\
\partial_{2} u_{3} \\
\partial_{1} u_{1}+\partial_{2} u_{2}
\end{array}\right)\left(x^{\prime}, x_{3}\right) d x_{3} .
\end{aligned}
$$

It is easy to see that $I_{\xi}\left(x^{\prime}\right)=0$ for $x^{\prime} \in \Gamma$ since $u\left(x^{\prime}, x_{3}\right)=0$ for $\left(x^{\prime}, x_{3}\right) \in$ $\Gamma \times(-L, L)$.

Next, we want to show that $I I_{\xi}\left(x^{\prime}\right)=0$ for $x^{\prime} \in \Gamma$. By denoting $\Gamma:=$ $S_{1} \cup S_{2}$, where $S_{1}, S_{2}$ are segments and $\overline{S_{1}} \cap \overline{S_{2}}=\left\{x_{0}^{\prime}\right\}$ is the corner point, we only need to demonstrate that $I I_{\xi}\left(x^{\prime}\right)=0$ on $S_{1}$. By choosing suitable boundary normal coordinates, without loss of generality, we may assume that $S_{1} \times(-L, L) \subset \operatorname{span}\left\{e_{1}, e_{2}\right\} \subset \mathbb{R}^{3}$ with its normal direction $\nu=e_{3}$. Here $\left\{e_{1}, e_{2}, e_{3}\right\}$ forms the standard orthonormal basis in $\mathbb{R}^{3}$. Recall that $u \in H_{l o c}^{2}\left(\mathbb{R}^{3}\right)$, then one has $\frac{\partial u_{j}}{\partial x_{k}} \in H_{l o c}^{1}\left(\mathbb{R}^{3}\right)$ for $j, k \in\{1,2,3\}$. Therefore, $\left.\frac{\partial u_{j}}{\partial x_{k}}\right|_{\Gamma \times(-L, L)}$ is a well-defined $L^{2}(\Gamma \times(-L, L))$-function in the trace sense. Since $u=0$ on $S_{1} \times(-L, L)$, we have $\frac{\partial u_{j}}{\partial x_{k}}=0$ for $j=1,2,3$ and $k=1,2$. Therefore, by using the boundary traction $\boldsymbol{T}_{\boldsymbol{\nu}} \boldsymbol{u}=0$ on $S_{1} \times(-L, L)$, and that $\mu>0, \lambda+2 \mu>0$ which follow from the assumptions, one can easily see that $\frac{\partial u_{j}}{\partial x_{k}}=0$ on $\Gamma \times(-L, L)$ for $j, k=1,2,3$. Similar arguments hold when $x^{\prime} \in S_{2}$, which proves that $I I_{\xi}\left(x^{\prime}\right)=0$ on $\Gamma$. This demonstrates Equation (3.9).

Remark 3.4. The boundary normal coordinates are useful in studying the inverse boundary value problem. The idea is based on the invariance of the elasticity system via change of variables. For example, in $[17,34]$, the authors utilized this technique to study the boundary determination for the isotropic elasticity system from the boundary measurements.

Proposition 3.5. Let $\Omega \subset \mathbb{R}^{3}$ be a bounded domain with $0 \in \partial \Omega$. Let $\theta_{m}$, $\theta_{M}$ be the number given by Proposition 3.2 and $\mathcal{K}$ be the cone defined by Equation (3.1). Suppose that $\Omega$ has an edge of opening angle $\theta_{M}-\theta_{m}$, that is, given an origin-centred ball $B \subset \mathbb{R}^{2}$ and there exists $L>0$ such that

$$
(B \times(-L, L)) \cap \Omega=(B \cap \mathcal{K}) \times(-L, L) .
$$

Given $\boldsymbol{f} \in C^{\alpha}\left((B \times(-L, L)) \cap \Omega ; \mathbb{C}^{3}\right)$ for some $\alpha \in(0,1)$, let $\boldsymbol{u} \in H^{2}((B \times$ $\left.(-L, L)) \cap \Omega ; \mathbb{C}^{3}\right)$ be a solution of $\mathcal{L} \boldsymbol{u}=\boldsymbol{f}$ in $B \cap \Omega$. Then

$$
\boldsymbol{u}=\boldsymbol{T}_{\boldsymbol{\nu}} \boldsymbol{u}=0 \text { on } B \cap \partial \Omega \text { implies that } \boldsymbol{f}(0)=0 .
$$

Proof. The proof is similar to the proof of [7, Proposition 3.5]. For the sake of completeness, we offer a detailed proof here. By the Sobolev embedding, we know that $H^{2}$ embeds to $C^{1 / 2}$ and we may assume $\alpha \leq 1 / 2$ without loss of generality. By Lemma 3.3, given any $\xi \in \mathbb{R}$, there is a $\boldsymbol{F}_{\xi} \in C^{\alpha}(\overline{B \cap \mathcal{K}})$, such that one can find a solution $\boldsymbol{U} \in H^{2}(B \cap \mathcal{K}) \cap C^{\alpha}(\overline{B \cap \mathcal{K}})$ fulfilling $\widetilde{\mathcal{L}} \boldsymbol{U}=\boldsymbol{F}_{\xi}$ in $B \cap \mathcal{K}$, where $\widetilde{\mathcal{L}}$ is defined by Equation (3.8). In addition, 
$\boldsymbol{U}=\boldsymbol{T}_{\boldsymbol{\nu}} \boldsymbol{U}=0$ on $B \cap \partial \mathcal{K}$, via Proposition 3.2, then we obtain $\boldsymbol{F}_{\xi}(0)=0$. Finally, recall that

$$
0=\boldsymbol{F}_{\xi}(0)=\int_{-L}^{L} e^{-i x_{3} \xi} \phi\left(x_{3}\right) \boldsymbol{f}\left(0, x_{3}\right) d x_{3},
$$

for any smooth cut-off functions $\phi\left(x_{3}\right) \in C_{c}^{\infty}((-L, L))$ and for any $\xi \in \mathbb{R}$. The Fourier inversion formula implies that $\boldsymbol{f}(0)=0$.

\section{Proof of Theorems}

In the end of this paper, we prove our theorems which stated in Section 1.

Proof of Theorem 1.1. Rellich's lemma for the Helmholtz equation (see e.g. Lemma 2.11 in [16]) and the unique continuation principle imply that $\boldsymbol{u}_{p}=$ $\boldsymbol{u}_{s}=0$ in the connected component of $\mathbb{R}^{n} \backslash \bar{\Omega}$ that reaches infinity. Hence $\boldsymbol{u}=0$ and $\boldsymbol{T}_{\boldsymbol{\nu}} \boldsymbol{u}=0$ on the boundary of the corner or edge. The claim follows from Proposition 3.2 or Proposition 3.5.

Proof of Theorem 1.2. By Rellich's lemma for the Helmholtz equation again and the unique continuation principle $\boldsymbol{u}_{p}=\boldsymbol{u}_{p}^{\prime}, \boldsymbol{u}_{s}=\boldsymbol{u}_{s}^{\prime}$ in $\mathbb{R}^{n} \backslash \overline{\Omega \cup \Omega^{\prime}}$. Assume $\Omega \not \subset \Omega^{\prime}$. Then by convexity there is a corner (2D) or edge (3D) point $x_{c} \in \partial \Omega \backslash \overline{\Omega^{\prime}}$. Since $\boldsymbol{u}=\boldsymbol{u}^{\prime}$ outside $\overline{\Omega \cup \Omega^{\prime}}$ we have $\boldsymbol{u}=\boldsymbol{u}^{\prime}$ and $\boldsymbol{T}_{\boldsymbol{\nu}} \boldsymbol{u}=\boldsymbol{T}_{\boldsymbol{\nu}} \boldsymbol{u}^{\prime}$ on $\partial \Omega$ near $x_{c}$. Set $\boldsymbol{w}=\boldsymbol{u}-\boldsymbol{u}^{\prime}$. We have

$$
\lambda \Delta \boldsymbol{w}+(\lambda+\mu) \nabla \nabla \cdot \boldsymbol{w}+\omega^{2} \boldsymbol{w}=\boldsymbol{f}
$$

in $\Omega$ near $x_{c}$ with $\boldsymbol{w} \in H^{2}$. Proposition 3.2 and Proposition 3.5 imply that $\varphi\left(x_{c}\right)=0$. But this is a contradiction since $\varphi \neq 0$ on $\partial \Omega$. Hence $\Omega \subset \Omega^{\prime}$. The same proof with $\Omega, \Omega^{\prime}$ switched shows that $\Omega^{\prime} \subset \Omega$. Hence $\Omega=\Omega^{\prime}$.

Next, let $x_{c}$ be a vertex $(2 \mathrm{D})$ or an edge point $(3 \mathrm{D})$ of $\partial \Omega=\partial \Omega^{\prime}$. If $\boldsymbol{w}=\boldsymbol{u}-\boldsymbol{u}^{\prime}$ then this time

$$
\lambda \Delta \boldsymbol{w}+(\lambda+\mu) \nabla \nabla \cdot \boldsymbol{w}+\omega^{2} \boldsymbol{w}=\boldsymbol{f}-\boldsymbol{f}^{\prime}
$$

in $\Omega$ with $\boldsymbol{w} \in H^{2}$. Rellich's lemma for the Helmholtz equation and the unique continuation principle for the Navier equations imply that $\boldsymbol{w}=0$ and $\boldsymbol{T}_{\boldsymbol{\nu}} \boldsymbol{w}=0$ on $\partial \Omega$ near $x_{c}$ in this case too. Proposition 3.2 and Proposition 3.5 imply $\boldsymbol{f}=\boldsymbol{f}^{\prime}$ at $x_{c}$.

Finally, we can prove the third main theorem in this paper.

Proof of Theorem 1.5. Set $\boldsymbol{f}=-\omega^{2} V \boldsymbol{v}$ and $\boldsymbol{u}=\boldsymbol{v}-\boldsymbol{w}$. In two and three dimensions $H^{2}$ embeds into $C^{\alpha}$ for $0<\alpha<1 / 2$ the latter of which we may assume. So $\boldsymbol{v}-\boldsymbol{w}$ is Hölder-continuous, and thus both $\boldsymbol{v}$ and $\boldsymbol{w}$ are too if either one is in $C^{\alpha}$. These functions satisfy

$$
\lambda \Delta \boldsymbol{u}+(\lambda+\mu) \nabla \nabla \cdot \boldsymbol{u}+\omega^{2} \boldsymbol{u}=\boldsymbol{f}
$$

with $\boldsymbol{u} \in H^{2}(\Omega), \boldsymbol{u}=\boldsymbol{T}_{\boldsymbol{\nu}} \boldsymbol{u}=0$ on $\partial \Omega$, and $\boldsymbol{f} \in C^{\alpha}(\bar{\Omega})$. Proposition 3.2 and Proposition 3.5 imply that $\boldsymbol{f}\left(x_{c}\right)=0$, so if $V\left(x_{c}\right) \neq 0$ then $\boldsymbol{v}\left(x_{c}\right)=0$ and since $\boldsymbol{v}=\boldsymbol{w}$ on $\partial \Omega$, so is $\boldsymbol{w}\left(x_{c}\right)=0$. 


\section{Appendix}

In the end of this paper, we demonstrate that a non-zero source function $\boldsymbol{f}$ may generate zero elastic far fields $\boldsymbol{u}_{s}^{\infty}$ and $\boldsymbol{u}_{p}^{\infty}$ in $\mathbb{R}^{3}$. Recall that the elastic far fields are given by

$$
\boldsymbol{u}_{s}^{\infty}(\boldsymbol{e})=\Pi_{\boldsymbol{e}^{\perp}}\left(\int_{\mathbb{R}^{3}} e^{-i \omega_{s} \boldsymbol{e} \cdot y} \boldsymbol{f}(y) d y\right), \boldsymbol{u}_{p}^{\infty}(\boldsymbol{e})=\Pi_{\boldsymbol{e}}\left(\int_{\mathbb{R}^{3}} e^{-i \omega_{p} \boldsymbol{e} \cdot y} \boldsymbol{f}(y) d y\right),
$$

for direction $e \in \mathbb{S}^{2}$, where $\Pi_{e}$ is the projection with respect to $e$. Now, simply take $\boldsymbol{f}:=e_{1} \chi_{B(0,1)}$, where

$$
\chi_{B(0,1)}= \begin{cases}1, & \text { in } B(0,1) \\ 0, & \text { otherwise }\end{cases}
$$

is the characteristic function and $e_{1}=(1,0,0)$.

After taking this special source function $\boldsymbol{f}=e_{1} \chi_{B(0,1)} \in L^{\infty}\left(\mathbb{R}^{3} ; \mathbb{C}^{3}\right)$, we want to find some suitable Lamé parameters $(\lambda, \mu)$ such that $\boldsymbol{u}_{s}^{\infty}(\boldsymbol{e})=0$ and $\boldsymbol{u}_{p}^{\infty}(\boldsymbol{e})=0$ for any direction $\boldsymbol{e} \in \mathbb{S}^{2}$. Moreover, the integral

$$
\int_{\mathbb{R}^{3}} e^{-i \omega_{s} e \cdot y} \chi_{B(0,1)} d y=\int_{\mathbb{R}^{3}} e^{-2 \pi i\left(\frac{\omega_{s}}{2 \pi} e\right) \cdot y} \chi_{B(0,1)} d y
$$

can be regarded as the Fourier transform of the characteristic function $\chi_{B(0,1)}$. By using the representation formula in [23, Appendix B.5], we have

$$
\int_{\mathbb{R}^{3}} e^{-i \omega_{s} e \cdot y} \chi_{B(0,1)} d y=\frac{J_{\frac{3}{2}}\left(\omega_{s}\right)}{\left(\frac{\omega_{s}}{2 \pi}\right)^{3 / 2}},
$$

where $J_{\frac{3}{2}}$ is the Bessel function of order $\frac{3}{2}$. Similarly, one also has

$$
\int_{\mathbb{R}^{3}} e^{-i \omega_{p} e \cdot y} \chi_{B(0,1)} d y=\frac{J_{\frac{3}{2}}\left(\omega_{p}\right)}{\left(\frac{\omega_{p}}{2 \pi}\right)^{3 / 2}} .
$$

Therefore, from Equations (5.1) and (5.2), having the elastic far fields $\boldsymbol{u}_{s}^{\infty}=\boldsymbol{u}_{p}^{\infty}=0$ can be reduced to seeking the Lamé parameters $(\lambda, \mu)$ such that

$$
J_{\frac{3}{2}}\left(\omega_{s}\right)=J_{\frac{3}{2}}\left(\omega_{p}\right)=0,
$$

where $\omega_{s}=\omega / \sqrt{\mu}$ and $\omega_{p}=\omega / \sqrt{\lambda+2 \mu}$. Let $A, B>0$ be any solutions of $J_{\frac{3}{2}}(t)=0$ with $B$ greater than $A$ large enough. Combining with Equation (5.3), without loss of generality, then we can choose

$$
A=\frac{\omega}{\sqrt{\lambda+2 \mu}} \text { and } B=\frac{\omega}{\sqrt{\mu}} \text {. }
$$

This is equivalent to taking the Lamé parameters as

$$
\lambda=\left(\frac{\omega}{A}\right)^{2}-2\left(\frac{\omega}{B}\right)^{2} \text { and } \mu=\left(\frac{\omega}{B}\right)^{2} .
$$

Note that by choosing $B$ large enough, the Lamé parameters $\lambda$ and $\mu$ satisfy the strong convexity condition Equation (1.1). Hence, for any frequency $\omega>0$, there are examples $(\lambda, \mu)$ and source $\boldsymbol{f}$ that is non-zero, but its far fields are zero. 
Acknowledgment. The authors appreciate Professor Ikehata and the anonymous referees for some useful comments to improve this work. Y.-H. Lin is partially supported by the Academy of Finland, under the project number 309963.

\section{REFERENCES}

[1] R. Albanese and P. B. Monk. The inverse source problem for Maxwell's equations. Inverse problems, 22(3):1023, 2006.

[2] S. R. Arridge. Optical tomography in medical imaging. Inverse problems, 15(2):R41, 1999.

[3] G. Bao, H. Ammari, and J. L. Fleming. An inverse source problem for Maxwell's equations in magnetoencephalography. SIAM Journal on Applied Mathematics, 62(4):1369-1382, 2002.

[4] G. Bao, C. Chen, and P. Li. Inverse random source scattering for elastic waves. SIAM Journal on Numerical Analysis, 55(6):2616-2643, 2017.

[5] G. Bao, S.-N. Chow, P. Li, and H. Zhou. An inverse random source problem for the Helmholtz equation. Mathematics of Computation, 83(285):215-233, 2014.

[6] C. Bellis, F. Cakoni, and B. B. Guzina. Nature of the transmission eigenvalue spectrum for elastic bodies. IMA Journal of Applied Mathematics, 78(5):895-923, 2013.

[7] E. Blåsten. Nonradiating sources and transmission eigenfunctions vanish at corners and edges. ArXiv e-prints, Mar. 2018.

[8] E. Blåsten, X. Li, H. Liu, and Y. Wang. On vanishing and localizing of transmission eigenfunctions near singular points: a numerical study. Inverse Problems, 33(10):105001, 24, 2017.

[9] E. Blåsten and H. Liu. On vanishing near corners of transmission eigenfunctions. J. Funct. Anal., 273(11):3616-3632, 2017.

[10] E. Blåsten and L. Päivärinta. Completeness of generalized transmission eigenstates. Inverse Problems, 29(10):104002, 22, 2013.

[11] E. Blåsten, L. Päivärinta, and J. Sylvester. Corners always scatter. Comm. Math. Phys., 331(2):725-753, 2014.

[12] F. Cakoni, D. Gintides, and H. Haddar. The existence of an infinite discrete set of transmission eigenvalues. SIAM J. Math. Anal., 42(1):237$255,2010$.

[13] F. Cakoni and H. Haddar. Transmission eigenvalues in inverse scattering theory. In Inverse problems and applications: inside out. II, volume 60 of Math. Sci. Res. Inst. Publ., pages 529-580. Cambridge Univ. Press, Cambridge, 2013.

[14] X. Cao, Y.-H. Lin, and H. Liu. Simultaneously recovering potentials and embedded obstacles for anisotropic fractional Schödinger operators. arXiv preprint arXiv:1712.00937, 2017.

[15] D. Colton, A. Kirsch, and L. Päivärinta. Far-field patterns for acoustic waves in an inhomogeneous medium. SIAM J. Math. Anal., 20(6):1472$1483,1989$.

[16] D. Colton and R. Kress. Inverse acoustic and electromagnetic scattering theory, volume 93 of Applied Mathematical Sciences. Springer-Verlag, Berlin, second edition, 1998. 
[17] M. V. De Hoop, G. Nakamura, and J. Zhai. Reconstruction of Lamé moduli and density at the boundary enabling directional elastic wavefield decomposition. SIAM Journal on Applied Mathematics, 77(2):520$536,2017$.

[18] A. El Badia and T. Nara. An inverse source problem for Helmholtz's equation from the Cauchy data with a single wave number. Inverse Problems, 27(10):105001, 2011.

[19] J. Elschner and G. Hu. Corners and edges always scatter. Inverse Problems, 31(1):015003, 2015.

[20] J. Elschner and G. Hu. Acoustic scattering from corners, edges and circular cones. Archive for Rational Mechanics and Analysis, 228(2):653690, 2018.

[21] A. Fokas, Y. Kurylev, and V. Marinakis. The unique determination of neuronal currents in the brain via magnetoencephalography. Inverse Problems, 20(4):1067, 2004.

[22] A. Friedman and V. Isakov. On the uniqueness in the inverse conductivity problem with one measurement. Indiana Univ. Math. J., 38(3):563$579,1989$.

[23] L. Grafakos. Classical Fourier analysis, volume 2. Springer, 2008.

[24] R. Griesmaier, M. Hanke, and J. Sylvester. Far field splitting for the Helmholtz equation. SIAM Journal on Numerical Analysis, 52(1):343$362,2014$.

[25] R. Griesmaier and J. Sylvester. Uncertainty principles for inverse source problems for electromagnetic and elastic waves. Inverse Problems, 34(6):065003, 2018.

[26] P. Hähner. On acoustic, electromagnetic, and elastic scattering problems in inhomogeneous media. PhD thesis, Universität Göttingen, Mathematisches Institut, 1998.

[27] G. Hu, M. Salo, and E. V. Vesalainen. Shape identification in inverse medium scattering problems with a single far-field pattern. SIAM J. Math. Anal., 48(1):152-165, 2016.

[28] M. Ikehata. Reconstruction of a source domain from the Cauchy data. Inverse Problems, 15(2):637-645, 1999.

[29] M. Ikehata. On reconstruction in the inverse conductivity problem with one measurement. Inverse Problems, 16(3):785-793, 2000.

[30] M. Ikehata and H. Itou. Extracting the support function of a cavity in an isotropic elastic body from a single set of boundary data. Inverse Problems, 25(10):105005, 2009.

[31] O. Y. Imanuvilov and M. Yamamoto. Global uniqueness in inverse boundary value problems for the Navier-Stokes equations and Lamé system in two dimensions. Inverse Problems, 31(3):035004, 2015.

[32] E. Lakshtanov and B. Vainberg. Applications of elliptic operator theory to the isotropic interior transmission eigenvalue problem. Inverse Problems, 29(10):104003, 19, 2013.

[33] P. Li. An inverse random source scattering problem in inhomogeneous media. Inverse Problems, 27(3):035004, 2011.

[34] Y.-H. Lin and G. Nakamura. Boundary determination of the Lamé moduli for the isotropic elasticity system. Inverse Problems, 33(12):125004, 
2017.

[35] W. McLean and W. C. H. McLean. Strongly elliptic systems and boundary integral equations. Cambridge university press, 2000.

[36] L. Päivärinta and J. Sylvester. Transmission eigenvalues. SIAM J. Math. Anal., 40(2):738-753, 2008.

[37] L. Robbiano. Spectral analysis of the interior transmission eigenvalue problem. Inverse Problems, 29(10):104001, 28, 2013.

[38] B. P. Rynne and B. D. Sleeman. The interior transmission problem and inverse scattering from inhomogeneous media. SIAM J. Math. Anal., 22(6):1755-1762, 1991.

Jockey Club Institute for Advanced Study, Hong Kong University of ScIence And Technology, Hong Kong SAR.

E-mail address: eemeli.blasten@iki.fi

Institute for Advanced Study, Hong Kong University of Science and Technology, Hong Kong \& Department of Mathematics and Statistics, University OF JYVÄSKYlä, FinLAND

E-mail address: yihsuanlin3@gmail.com 